\title{
Editorial
}

\section{Evidence-Based or Evidence-Biased: The Need to Re-appraise and Re-align Levels of Information with Stakeholder Values}

Mark V. Boswell, MD, PhD¹, James Giordano, $\mathrm{PhD}^{2}$

$\square$ ne of the incentives of the incoming presidential administration is consideration of healthcare reform(s), and certainly, pain medicine would be impacted by any such change(s). We feel that this will be beneficial, as pain medicine cannot remain static, but must incorporate new information, knowledge, and capabilities, and be empowered in these pursuits. One of the true challenges is how to utilize the groundswell of research fostered by the Decade of Pain Control and Research initiative, so as to maximize the intent of governmental policies toward, and realities of, providing safe and effective pain care, in ways that support the public good.

President Obama has recognized the need to establish a medical system that both decreases the effects of disease and illness, and facilitates preventive strategies and tactics. Toward these ends, he has proposed evidence-based analyses of treatments and outcomes, as well as patient and physician choices, needs, and resources (1). Such acknowledgment of both supply and demand variables can be seen as a positive direction for pain medicine, and we believe that this re-organization is necessary in light of the past years' scientific developments, number of viable, emergent techniques and technologies, and the contingencies that these advances incur for both commutative and distributive equity in the allocation of the goods and services that should be rendered in pain care.

Demand-side variables consider several patientbased factors - not only the those specifically dictated by a particular pain diagnosis and/or nosology, but also other biological, psychological, and socio-economic constraints - and the values that are related to each (2). Thus, to a large extent, clinical equipoise is a dialectic process involving the (clinical) values of the physician, and values patients place upon different approaches to their care. This interactive decisional process engages the physician as a steward of knowledge to discern what types of care are best suited to each patient's medical condition, limitations, resources, goals, and values. Different types and levels of evidence may be used to develop and support these value sets (3-5). To be sure, there is some common evidentiary norm - perhaps patient safety - that ultimately dictates choice(s) in medical decision-making. However, when examining and assessing certain benefits versus other (perhaps less absolute) risks and burdens, it seems that such comparisons are relative to the value(s) that physicians and patients place upon certain outcomes and/or contingencies of care.

Therefore, we question whether evidence, and/ or evidence-hierarchies are, or can be, a "one-sizefits-all" construct. Given the heterogeneity of patient (genotypic, phenotypic. and hence bio-psychosocial) variables, it may be that relative value of any/all evi-

From: ${ }^{1}$ Messer-Racz International Pain Center, Texas Tech University Health Sciences Center, Lubbock, TX; ${ }^{2}$ Georgetown University Medical Center, Washington, DC; and Center for Neurotechnology Studies Potomac Institute for Policy Studies, Arlington, VA.

Dr. Boswell is Professor and Chair, Dept of Anesthesiology, MesserRacz International Pain Center, Texas Tech University Health Sciences Center, Lubbock, TX.

Dr. Giordano is Samueli-Rockefeller Professor, Dept of Medicine and Neurosciences, and Center for Bioethics, Georgetown University Medical Center, Washington, DC; and Senior Fellow and Director, Center for Neurotechnology Studies, Potomac Institute for Policy Studies, Arlington VA.

Address correspondence: Prof. J. Giordano, PhD Depts of Medicine and Neurosciences Georgetown University Medical Center 4000 Reservoir Rd, Bldg D Washington, DC 20057

E-mail: jg353@georgetown.edu

Disclaimer: Supported by L.S. Rockefeller Trust (JG), AAPM Visiting Professorship (JG). 
dence to specific "stakeholders" in the clinical relationship (and its course and outcomes) must be taken into account when both 1) examining how and what (types of) evidence is right, and 2) how any such evidence can and should be utilized to maximize the "good" of both the patient (as the stakeholder who often has the most "at stake" in the clinical encounter), and the profession and practice of medicine as the nexus for the therapeutic and moral fiduciary.

In this issue of Pain Physician, Cindy Crawford, Mylene Huynh, Alyson Kepple, and Wayne Jonas (6) illustrate the issues, and problems that are inherent to, and arise from, attempting an evidence-based comparison of types of treatments for headache, a condition commonly seen in the practice of pain medicine. This is a worthwhile area of inquiry; the information provided by Crawford et al (6) adds to that of the Cochrane Library (7), and given the epidemiology of headache, and the variety of possible treatments that may be employed, this review is of obvious worth to the pain physician. However, we feel that the more important point made by the authors relates to the "state-ofthe-field," not simply of complementary therapeutics (although the authors make several noteworthy observations in this regard), but of evidence-based practice (or evidence-based medicine, EBM) as a system of clinical information evaluation. Recent reviews of evidence-based pain medicine by Manchikanti (7) and Manchikanti et al (8) have also explicated many of these issues, and when taken together, these papers present a critique of the values and validity of those methods of experimental design, and data analysis and interpretation that fundamentally dictate the scope, nature, and trajectory of much of practice of pain medicine (if not medicine, at large).

For example, in discussing how CAM-based approaches to headache may be evaluated against more "mainstream" therapeutics, Crawford et al (6) address how the Jadad scale, while popular in the past, may not be a viable means of assessing and ranking such studies, given its inherent limitations to appreciate the distinctions between various research protocols that may or may not be possible in certain clinical settings. These limitations have been recognized by both the Cochrane group and the AHRQ. Crawford and colleagues (6) are convinced that the state of the literature is such that it is possible to make broad generalizations about not only the apparent distinctions in quality between CAM and mainstream medical reporting, but also about the relative value skewing that can occur as a consequence of over-regarding various types of studies and/or data. As a result, durable biases for or against whole systems and categories of treatment may be fostered, when in fact, other measures of study and data quality (and worth) might be better suited to provide a more accurate representation of the validity and value of particular evidence. Thus, while it is likely that in some cases, "mainstream" approaches may indeed be more effective than CAM, the problems in reporting and judging evidence in support of this claim may make any head-to-head comparison factually difficult. Similar difficulties are frequently encountered when trying to evaluate the evidence illustrating the effectiveness of pharmacological versus non-pharmacological treatments (not only of headache, but also for musculoskeletal or certain forms of discogenic spinal pain), where any negative results (particularly in those trials conducted by pharmaceutical firms) might not be reported, thereby imparting a positive skewing of the data, that would be fortified by the judged "level of evidence" (i.e., a multi-center, randomized, controlled trial, RCT). In other words, both Crawford et al (6) and Manchikanti and co-workers $(8,9)$ have provided review and critique of what has become something of a world view (i.e., a Weltanschauung) in healthcare - evidence-based medicine (EBM) - and in this way illustrate its strengths, limits, and weaknesses.

We opine that these criticisms do not dispel or disarm EBM, but rather suggest that EBM must acknowledge a changing epistemology, engage new knowledge of the bio-psychosocial complexity of patients, and revise methods toward developing and sustaining a progressive, current paradigm for the valuation of information. This is essential given the disparity between knowledge and clinical skills, and the necessity to weigh abundant information in the process of clinical decision-making. By definition, EBM entails an integration of (best) research evidence, clinical expertise, and patient needs. None of these variables are value free. Simple acknowledgment of this fact demands an approach that can and should appreciate the relative value of different evidence to distinct stakeholders in the course and outcome of clinical decisions and practice(s).

What then, constitutes "best" research evidence? The reviews by Crawford et al (6) and Manchikanti et al demonstrate $(8,9)$ considerable variability in techniques, protocols, and methods, and it is often difficult to make prima facie determinations of whether a par- 
ticular research approach was best suited to maximally elicit specific outcomes. Similarly, clinical expertise is not a monolithic entity. It too reflects particular values that may dictate physicians' decisional process and/or predispositions towards certain clinical approaches and/or types of care. Moreover, clinical expertise is reliant, at least in part, upon the physician's ability to intuit the benefits, burdens, and risks of various treatments relative to both a patient's diagnosis (i.e., the pathologic condition), and its impact upon the patient's lived experience and expectations (i.e., illness effects). Each of the dimensions of EBM are embedded with stakeholder values, and thus the goal of EBM is to utilize various levels of information, as relevant to these values, to pose an answer to the prudential questions of "what can be done," and most importantly, "what should be done to treat this patient" (10). Hence, the fundamental process of EBM compels evaluation of the "best" evidence for the circumstances relevant to both the patient and the integrity of clinical care. This involves a multi-step process. First is a critical appraisal of any evidence for its validity. Second, is the integration of this appraisal with a given patient's specific biological condition, circumstances, and goals and values. Last, is the need to evaluate the evidence and the process, itself. Namely, does the evidence match the variables of a particular case with sufficient alignment so as to justify use. In light of this, we opine that EBM is a casuistic process that is reliant upon the practical wisdom of the physician to uphold the primacy of the patient's best interest. While this process may be enacted in its entirety, at least to some extent in every clinical case, more common clinical problems tend to require evidence review and appraisal, less frequent clinical problems may require the integration of information from larger data banks (e.g. Cochrane reviews; meta-analyses, etc.) and rare clinical problems can require either replication of some other clinical report (including perhaps $\mathrm{N}=1$ studies,) or may necessitate a unique therapeutic approach that is based upon rational use of related information and clinical intuition and skill.

The limitations of EBM reflect the core constraints of much of medical science in practice. These include 1) lack of consistent scientific evidence; 2 ) difficulties in applying techniques studied in controlled settings to the actual care of individual patients; and 3) limited time and resources for effective review of available and best evidence. To date, third party payers have been a driving force in sustaining EBM, albeit primarily as a means of cost containment. However, in reality EBM may not decrease healthcare costs that are necessary in the treatment of individual patients. When evidence acknowledges and appreciates the resonance of physician and patient values so as to support the appropriate use of both high- and lowtech treatments as best suit the needs of the patient, then as matter of fact, the best treatment might not be "the cheapest," but there is a high likelihood that it will be the most prudent choice, and therefore the least wasteful of medical resources (11-13). We argue that if conducted in this way, EBM is not "cookbook" medicine, but rather becomes a viable tool to enable soundly practical casuistry.

Defining prudence as practical wisdom necessitates that some form of guidance must be available and utilized to navigate the complexities offered by each clinical encounter and its constituent decisions. Our hope is that President Obama's directive to develop healthcare practices based upon the best available evidence will 1) recognize how values mandate that such evidence be tiered relative to particular stakeholders, and 2) incorporate these evidence hierarchies into clinical guidelines and healthcare policy. If done in this way, Mr. Obama's model of evidence-based healthcare may foster the translation of scientific research to medical practice, and thus, facilitate improvement in both. Such explicit goals would be important when formulating policies that 1) direct and support basic and clinical research; 2) develop and sustain databanks and administrative infrastructure for the evaluation of (various types and levels of) evidence; and 3) empower and subsidize healthcare programs that utilize appropriate evidence in clinical practice. We claim that in order to best develop such guidelines and policies, government must collaborate with physician organizations to produce and evaluate scientific evidence that can best accommodate patients' needs. Extant and future considerations of any/all evidence that will be utilized in clinical practice might require some reappraisal of how qualitatively different types, forms, and levels of evidence might be used by different stakeholders, and how evidence-stakeholder interactions might affect the relative strength of any clinical recommendations.

In conclusion, we commend President Obama's intentions and efforts to develop a more effective "evidence-based" healthcare system, and we are hopeful that any meaningful attempt at this enterprise will reassess EBM as process and purpose; as Crawford 
et al (6) and Manchikanti and colleagues $(8,9)$ have shown, EBM is certainly not value-free and not without flaw(s). Yet, EBM is, and can be, evermore useful. The fundamental revision toward this utility involves appreciation of what factors and values determine "best" evidence. Clearly, this is an iterative endeavor that will improve pain medicine by making sound, effective patient-centered pain care a reality.

\section{Acknowledgments}

This work was supported in part by a grant from the Laurance S. Rockefeller Trust (JG), an American Academy of Pain Medicine National Visiting Professorship (JG), and funding from the Department of Anesthesiology and Messer-Racz International Pain Center, Texas Tech University Health Sciences Center (MVB, JG). The authors acknowledge the assistance of Sherry Loveless and Holly Long on preparation of this manuscript.

\section{References}

1. Candidate Commentaries. Modern Healthcare 2007; 11: 12-21.

2. Satz, AB. Toward solving the health care crisis: The paradoxical case for universal access to high technology. Yale J Health Policy, Law, and Ethics. 2008; VIII:1:93-143.

3. Goodman, KW. Ethics and EvidenceBased Medicine: Fallibility and Responsibility in Clinical Science. Cambridge University Press, Cambridge, MA, 2003.

4. Entwistle V, O'Donnell M. Evidencebased health care: What roles for patients? In: Adrian E and Elwyn G (eds). Evidence-Based Patient Choice: Inevi table or Impossible? Oxford University Press, Oxford, UK, 2001, p. 34-52.

5. Jonas WB. Evidence, ethics and the evaluation of global medicine. In: Callahan D (ed). The Role of Complemen tary and Alternative Medicine: Accomodating Pluralism. Georgetown Uni- versity Press, Washington, DC, 2002, p. 122-141.

6. Crawford C, Huynh M, Kepple A, Jonas W. Assessment of the quality of research studies of conventional and alternative treatment(s) of primary headache. Pain Physician. 2009; 12:263272.

7. Bronfort G, Nilsson N, Haas M, Evans R, Goldsmith $\mathrm{CH}$, Assendelft WJJ, Bouter LM. Non-invasive physical treatments for chronic/recurrent headache. Cochrane Database of Systematic Reviews 2004, Issue 3. Art. No.: CDo01878. DOI: 10.1002/14651858.CDo01878.pub2.

8. Manchikanti L. Evidence-based medicine, systematic reviews, and guidelines in interventional pain management, Part 1: Introduction and general considerations. Pain Physician 2008; 11:161-186.

9. Manchikanti L, Hirsch JA, Smith HS. Evidence-based medicine, systematic re- views, and guidelines in interventional pain management: Part 2: Randomized controlled trials. Pain Physician 2008; 11:717-773.

10. Pellegrino ED. The healing relationship: Architectonics of clinical medicine. In: Shelp EE (ed). The Clinical Encounter: The Moral Fabric of the Physician-Patient Relationship. Reidel, Boston, MA, 1983.

11. Giordano J. Technology in pain medicine: Research, practice, and the influence of the market. Prac Pain Management 2008; 8;56-59.

12. McClellan M, Kessler D. A global analysis of technological change in health care: The case of heart attacks. Health Aff 1999; 18:250-253.

13. Jost TS. The American difference in health care costs: Is there a problem? Is medical necessity a solution? St. Louis Univ Law J 1999; 1:43. 